\title{
Enteral tube feeding for people with severe dementia (Protocol)
}

Davies N, Barrado-Martin Y, Rait G, Fukui A, Candy B, Smith CH, Manthorpe J, Moore KJ, Vickerstaff V, Sampson EL

Davies N, Barrado-Martin Y, Rait G, Fukui A, Candy B, Smith CH, Manthorpe J, Moore KJ, Vickerstaff V, Sampson EL. Enteral tube feeding for people with severe dementia.

Cochrane Database of Systematic Reviews 2019, Issue 12. Art. No.: CD013503.

DOI: 10.1002/14651858.CD013503.

www.cochranelibrary.com 
TABLE OF CONTENTS

HEADER 1

ABSTRACT

BACKGROUND

OBJECTIVES

METHODS

ACKNOWLEDGEMENTS

REFERENCES

APPENDICES

CONTRIBUTIONS OF AUTHORS

DECLARATIONS OF INTEREST

SOURCES OF SUPPORT

\section{1}

2

3

3

7

8

10

11 
[Intervention Protocol]

\section{Enteral tube feeding for people with severe dementia}

Nathan Davies ${ }^{1}$, Yolanda Barrado-Martin 1 , Greta Rait ${ }^{1}$, Akiko Fukui², Bridget Candy ${ }^{3}$, Christina H Smith4, Jill Manthorpe ${ }^{5}$, Kirsten J Moore ${ }^{3}$, Victoria Vickerstaff3, Elizabeth L Sampson ${ }^{3}$

${ }^{1}$ Centre for Ageing Population Studies, Research Department of Primary Care and Population Health, University College London, London, UK. ${ }^{2}$ Medical School, St George's, University of London, London, UK. ${ }^{3}$ Marie Curie Palliative Care Research Department, Division of Psychiatry, University College London, London, UK. ${ }^{2}$ Division of Psychology and Language Sciences, University College London, London, UK. ${ }^{5}$ NIHR Health and Social Care Workforce Research Unit, The Policy Institute, King's College London, London, UK

Contact address: Elizabeth L Sampson, Marie Curie Palliative Care Research Department, Division of Psychiatry, University College London, 6th Floor, Maple House, 149 Tottenham Court Road, London, W1T 7NF, UK. e.sampson@ucl.ac.uk.

Editorial group: Cochrane Dementia and Cognitive Improvement Group

Publication status and date: New, published in Issue 12, 2019.

Citation: Davies N, Barrado-Martin Y, Rait G, Fukui A, Candy B, Smith CH, Manthorpe J, Moore KJ, Vickerstaff V, Sampson EL. Enteral tube feeding for people with severe dementia. Cochrane Database of Systematic Reviews 2019, Issue 12. Art. No.: CD013503. DOI: 10.1002/14651858.CD013503.

Copyright ( 2019 The Cochrane Collaboration. Published by John Wiley \& Sons, Ltd.

\section{A B S T R A C T}

This is a protocol for a Cochrane Review (Intervention). The objectives are as follows:

To evaluate the outcome of enteral tube feeding for people with severe dementia who develop problems with eating and swallowing or whose intake of food and fluid is reduced due to progression of the dementia. 


\section{B A C K G R O U N D}

\section{Description of the condition}

Dementia is a syndrome of cognitive decline that leads to impairment in two or more cognitive domains, including memory, executive functions, attention, language, and visuospatial abilities. The cognitive impairment causes functional decline, interfering with an individual's ability to conduct activities of daily living (WHO 2018). Most dementias are caused by progressive neurodegenerative conditions. The most common cause of dementia is Alzheimer's disease, followed by vascular dementia (WHO 2012). Mixed dementias (with more than one underlying cause) are also common (Alzheimer's Disease International 2009; Livingston 2015). The progression of dementia is highly variable between individuals and is often complicated by other health conditions. Prognosis is difficult to predict. Median survival has been estimated at 4.1 years (Xie 2008). Survival times from the point of diagnosis in primary care are estimated to be 6.7 years in those aged 60 to 69 years, dropping to 1.9 years in those diagnosed aged 90 years and over (Rait 2010). Dementia is associated with the fastest rise in serious health-related suffering and the number of people with dementia requiring palliative care is expected to increase 4 -fold by 2060 (Sleeman 2019).

Currently an estimated 50 million people live with dementia worldwide, and this is expected to rise to 152 million in 2050 (WHO 2019). In 2015 there were an estimated 9.9 million new cases of dementia globally each year, equating to a new diagnosis every $3.2 \mathrm{sec}-$ onds (Alzheimer's Disease International 2015). The numbers of people living with dementia are higher in East Asia than anywhere else in the world (9.8 million), followed by Western Europe (7.5 million), South Asia (5.1 million), and North America (4.8 million) (Alzheimer's Disease International 2015). This trend suggests that by 2050 there will be a $264 \%$ increase of people living with dementia in low-income countries, $227 \%$ in upper middle-income countries, $223 \%$ in lower middle-income countries and $116 \%$ in high-income countries (Alzheimer's Disease International 2015; Matthews 2013).

People with dementia become increasingly dependent on those around them, usually family members, for support with activities of daily living. In the early stages, most types of dementia are characterised by changes in memory and other higher cognitive functions, such as communication (e.g. difficulty finding the right words), disorientation, and difficulty with household tasks. Symptoms progress differently in each person but generally the person with dementia will become increasingly forgetful and need help with personal care and communication. He or she may also develop changes in behaviour such as disinhibition or aggression, or psychiatric symptoms such as hallucinations. In the severe stages, someone with dementia becomes completely dependent on others. Common symptoms in severe dementia include immobility, double incontinence, agitation, and pain (Sampson 2018). Problems with eating are almost universal; cohort studies have found up to $86 \%$ of people with advanced dementia will have difficulties with eating or swallowing in the last 6 months of life (Mitchell 2016). Swallowing difficulties may be more problematic in vascular dementia where there is a greater risk of stroke but that there is little literature on the prevalence of swallowing difficulties by dementia type.

\section{Description of the intervention}

Difficulties with eating are common among people with dementia (Mitchell 2016; Sampson 2018); this may be noticed before a formal diagnosis is made. It can be hard for people with dementia to maintain their weight or to drink enough. During the early stages of dementia, problems may be caused by the person forgetting to eat or becoming disorientated at mealtimes. There may be changes in how food is perceived, including altered taste and smell of food, which may make it unappetising (Kai 2015). In the later stages of dementia, people may develop physical difficulties with the act of swallowing, for example failing to manage the food properly once it is in the mouth (oral phase dysphagia), or food or drink going down the "wrong way" into the lungs (aspiration) when swallowing (pharyngeal phase dysphagia) (Volkert 2015). Whether or not to intervene by feeding artificially via an enteral tube (passed through the mouth or stomach wall) is a common clinical dilemma facing those who care for people with severe dementia (Brooke 2015; Davies 2016; Davies 2018; De 2019; Mathew 2016; Watt 2019).

\section{How the intervention might work}

In this Cochrane Review we define "enteral tube feeding" as the administration of food via a nasogastric tube, via a percutaneous endoscopic gastrostomy (PEG) tube or via jejunal post-pyloric feeding. In nasogastric feeding, the tube is passed through the nose and down to the stomach via the oesophagus. In PEG the feeding tube is passed through an endoscope, down the oesophagus and into the stomach. It is then guided out through an incision in the abdominal wall. Jejunal post-pyloric feeding is a method of feeding directly into the small bowel where the feeding tube is passed through the mouth, oesophagus, stomach, and into the jejunum. We will exclude intravenous administration of fluids from this review because this is more commonly used as a short-term intervention during episodes of acute physical illness.

\section{Why it is important to do this review}

Difficulties with eating are challenging for all those involved, including the person with dementia, their family, and health and social care staff. Family carers report a lack of information on what to expect during the later stages of dementia and a need for knowledge about end-of-life care (Davies 2014; Davies 2017), particularly around nutrition and hydration (Papachristou 2017). Enteral tube feeding may be perceived to be a method of managing malnutrition and weight loss, reducing the risk of aspiration associated with oral feeding, preventing pressure ulcers, or simply increasing quality of life and comfort. Alternatively, enteral tube feeding may be a temporary intervention while managing intercurrent physical illness, such as pneumonia and other infections where control of the swallowing reflex may be temporarily impaired. However, how these decisions are made and the use of these interventions varies according to patient need, the clinical team, culture, country, family carer preference, and the presence of advance care plans.

The decision to intervene with enteral tube feeding is complex and emotive (Braun 2005; Volkert 2015). In most societies, providing food or feeding for someone is seen as a sign of care and has 'symbolic significance' (Volkert 2015). Not providing food or nutrition can be seen as a symbol of neglect, allowing the person to go hungry or starve to death (Hoefler 2000). There is additional complexity because people with severe dementia lack the capacity to make this decision for themselves; depending on the laws in their coun- 
try, it may then be made in their 'best interests' by others. The use of enteral tube feeding has implications for the dignity and personhood of the individual. Professionals and family carers may have conflicting concerns regarding using tube feeding. They may think it can prolong life, increase nutritional status, and prevent complications (Punchik 2018; Snyder 2013). Families may perceive that a lack of enteral tube feeding when someone cannot swallow or eat causes starvation, and not intervening may be considered a form of euthanasia (Gil 2018).

The evidence about effects of enteral tube feeding is contradictory and long-term use of this intervention is not encouraged. Previous studies, including the earlier Cochrane Review, found no evidence that enteral tube feeding was effective for increasing survival, improving quality of life, improving nutritional parameters (measured with blood tests), or decreasing pressure sores (American Geriatrics Society 2014; Sampson 2009).

There is evidence that enteral tube feeding may increase mortality and morbidity, and reduce quality of life (Cintra 2014). Studies have demonstrated that enteral tube feeding is associated with increased discomfort, aspiration pneumonia, and worsening of urinary and faecal incontinence (Ciocon 1988; Finucane 1996; Odom 2003). Incontinence increases pressure sore risk and there is also evidence that enteral tube feeding increases the risk of pressure sores and does not aid the healing process (Teno 2012).

PEG surgical procedures are invasive, may cause distress and discomfort, and risk bowel perforation, localised bleeding, inflammation, and infection. A study of deaths following PEG tube insertion in 719 people with predominantly neurological diagnoses in the UK found that $2 \%$ died on the day the PEG was inserted and $43 \%$ died within 7 days. In $19 \%$ of cases the procedure was regarded as futile by an expert panel (Johnston 2008). PEG can be particularly problematic for someone with dementia who may not recognise the device, may be distressed by it and may attempt to remove the tube. To reduce this risk, patients may need to be restrained either physically (e.g. through the use of mittens) or chemically (e.g. through sedation). Others, however, have argued that the evidence of harm is unclear and that PEG may not increase mortality in people with dementia (Brooke 2015).

Although there are increasing numbers of recommendations that highlight the lack of evidenced benefit and potential risks for enteral tube feeding (American Geriatrics Society 2014; Sampson 2009; van der Steen 2014), debate continues on this issue (van der Steen 2014). An absence of evidence does not mean the intervention may not be effective or appropriate for some people, and ruling out this intervention for all people with severe dementia prevents clinicians and patients from delivering individually-tailored care (Regnard 2010). Trends in practice in this area vary in different parts of the world. For example, there is decreasing use of enteral tube feeding in the UK and the USA (Mitchell 2016), but there appears to be increasing use in other countries, such as Taiwan (Chang 2016).

It should be acknowledged that swallowing difficulties may not always indicate the terminal stages of dementia. Over $91 \%$ of people with dementia will be living with at least one co-morbid condition (Browne 2017), including stroke (Bunn 2014), which could affect swallowing abilities in the earlier stages of dementia. Acute illness, for example infections such as pneumonia, may also temporarily impair swallowing ability.
The earlier Cochrane Review is now over 10 years old (Sampson 2009). It continues to be cited in policy and guidance documents, including a decision aid developed as part of the dementia guideline developed in 2018 by the National Institute for Health and Care Excellence (NICE) in the UK (NICE 2018). It is important that the review is updated. New studies have been published since the original review was written and an update will ensure the conclusions remain helpful in clinical practice.

\section{O B JECTIVES}

To evaluate the outcome of enteral tube feeding for people with severe dementia who develop problems with eating and swallowing or whose intake of food and fluid is reduced due to progression of the dementia.

\section{METHODS}

\section{Criteria for considering studies for this review}

\section{Types of studies}

We will include a range of controlled comparison studies: randomised controlled trials (RCTs), controlled trials, controlled before-and-after studies, and interrupted time series studies. We will include studies that compare enteral tube feeding with no intervention or 'usual treatment or care' or with another active intervention, such as finger food. Studies may be in any healthcare setting (including acute hospitals), residential settings (nursing, residential homes or care homes), or in participants' own homes. We will include studies published in any language.

Some researchers and other stakeholders with interests in this area may consider controlled evaluation to be inappropriate or unethical in such a patient group. We are aware that for ethical reasons researchers may choose to explore effects without a control group. We will therefore include observational studies, but we will analyse data from such studies separately and with caution due to the inherent weakness of such designs.

\section{Types of participants}

We will include adult participants of any age and gender, with a clinical diagnosis of primary degenerative dementia of any cause made according to validated diagnostic criteria such as Diagnostic and Statistical Manual 4th edition (DSM-IV) or International Classification of Diseases 10th edition (ICD-10) (APA 2000; WHO 1993). We will include participants with severe cognitive and functional impairment. This will be defined by a recognised and validated tool (e.g. stage 7A or above on the Functional Assessment stage (FAST) tool (Reisberg 1994), Clinical Dementia Rating (CDR) Scale score of 3 , Cognitive Performance Scale score 5 or 6 ) or by clinical assessment - and who are reported to have poor nutritional intake. We will measure poor nutritional intake using clinical tools (i.e. Malnutrition Universal Screening Tool (MUST)). We will include studies reporting on participants with a diagnosis of Alzheimer's disease, vascular dementia, mixed dementia, Lewy body dementia, or fronto-temporal lobar degeneration (FTLD). Patients may live at home or in any health or care setting.

\section{Types of interventions}

We will include studies that evaluate the effectiveness of enteral tube feeding via a nasogastric tube, via a tube passed by PEG, or via jejunal post-pyloric feeding to deliver nutrition. We will exclude 
studies of oral dietary supplementation. Comparators may include no intervention/standard care, waiting list, or enhanced standard care, such as an intervention to promote oral intake including a textured modified diet.

\section{Types of outcome measures}

\section{Primary outcomes}

This review will focus on three primary outcomes:

- Survival time (measured by the time-to-event post-intervention).

- Quality of life (measured by a recognised and validated quality of life scale or tool, such as the quality of life in late-stage dementia (QUALID) scale or the Dementia Quality of Life scale (DemQoL).

- Presssure ulcers.

\section{Secondary outcomes}

We will include as secondary outcome measures:

- Pain and comfort if these are measured with validated scales.

- Mortality.

- Change in behavioural and psychological symptoms of dementia (indicators of distress) measured using a validated scale (e.g. the Neuropsychiatric Inventory (NPI)).

- Improvement of nutritional parameters (e.g. albumin levels).

- Family carer outcomes such as depression, anxiety, carer burden, satisfaction with care, or increased sense of competence measured using validated scales (e.g. Hospital Anxiety and Depression Scale, Beck Depression Inventory, Zarit Burden Interview, Sense of Competence Questionnaire, Satisfaction with care at the end of life in dementia (SWC-EOLD)).

- Harm-related outcomes (adverse events) such as:

* aspiration pneumonia

* gastrointestinal and urinary (i.e. constipation, reflux, urinary/faecal incontinence)

* local bleeding;

* infections;

* systemic (i.e. fluid imbalance or overload

* feeding tube problems (i.e. blocking or need for tube to be resited, if follow-up time is sufficient).

\section{Search methods for identification of studies}

\section{Electronic searches}

We will search ALOIS (www.medicine.ox.ac.uk/alois), which is the Cochrane Dementia and Cognitive Improvement Group's Specialised Register.

ALOIS is maintained by the Information Specialists of the Cochrane Dementia and Cognitive Improvement Group and contains studies in the areas of dementia (prevention and treatment), mild cognitive impairment, and cognitive improvement. The studies are identified from:

- Monthly searches of a number of major healthcare databases: MEDLINE, Embase, CINAHL, PsycINFO, and LILACS.

- Monthly searches of a number of trial registers: ISRCTN; UMIN (Japan's Trial Register); the World Health Organization (WHO) portal (which covers ClinicalTrials.gov; ISRCTN; the Chinese Clinical Trials Register; the German Clinical Trials Register; the Iranian Registry of Clinical Trials; the Netherlands National Trials Register; and others).

- Quarterly searches of the Cochrane Library's Central Register of Controlled Trials (CENTRAL).

- Six-monthly searches of a number of grey literature sources: ISI Web of Knowledge Conference Proceedings; Index to Theses; Australasian Digital Theses.

To view a list of all sources searched for ALOIS, see About ALOIS on the ALOIS website.

Details of the search strategies used for the retrieval of reports of trials from the healthcare databases, CENTRAL, and conference proceedings can be viewed in the 'Methods used in reviews' section within the editorial information about the Cochrane Dementia and Cognitive Improvement Group.

We will perform additional searches of many of the sources listed above to ensure that we capture non-RCTs, controlled before-andafter studies, and interrupted time series studies. The MEDLINE search strategy we will use is in Appendix 1.

\section{Searching other resources}

We will contact experts in the field by posting calls for evidence to identify any further trial evaluations that were not identified in the citation databases searches. We will also search the conference proceedings of the European Association for Palliative Care, International Psychogeriatrics Association Conference, BAPEN, and the Association of British Neurologists. We will screen reference lists of included articles and will track citations.

\section{Data collection and analysis}

\section{Selection of studies}

In accordance with the defined inclusion criteria, we will screen citations in duplicate independently. Three review authors (YBM/AF/ ND) will screen all citations and will check each other's screening decisions. We will classify the citations into three groups: 'exclude', 'potentially relevant' or 'unsure'. We will exclude papers classified by two review authors as 'exclude'. We will retrieve the full-text versions of all 'potentially relevant' and 'unsure' citations for definitive assessment of eligibility. One author (YBM/AF) will independently screen the full texts for a comprehensive assessment against the inclusion criteria. A second author will check judgements (ND/ELS). We will resolve any disagreements through discussion. When required we will consult with the wider review author team. We will document justification for excluding studies at this stage, and will illustrate the study selection process in a PRISMA diagram.

\section{Data extraction and management}

We will design a data extraction form for the review. Where possible, we will obtain the following information for each included study:

- The number of eligible patients, number randomised if an RCT, and reasons why patients were not included in the study.

- The number of patients evaluated at follow-up(s) and what the follow-up time points were. 
- Patient characteristics including age, sex, co-morbidities, diagnosis and type of dementia, advance decision or proxy decision maker status, type of health care or community setting, stage of disease when enteral nutrition or other intervention was considered, and reason for enteral nutrition or other intervention.

- Study design features, for example if an RCT on masking, whether parallel or cross-over, features of randomisation, and sample size calculation.

- Enteral nutrition intervention including dosage, duration and mode including the need to restrain the patient.

- Comparison intervention including duration and mode.

- Outcome data at all time points including how it was measured, and the mean or categorical scores of the primary and secondary outcomes.

Specifically for non-randomised studies we will follow guidance from the Cochrane Handbook for Systematic Reviews of Interventions (Reeves 2019). We will consult the Cochrane Non-Randomised Studies Methods Group (NRSMG). Where possible we will also extract:

- Data on confounding factors considered and methods used to control for confounding. We will use the ROBINS-I tool as a template for this information (Sterne 2019).

- Comparability of groups on confounding factors considered.

- Data about multiple effect estimates (both unadjusted and adjusted estimates, if available)

- For study design we will use the checklist from the Cochrane NRSMG (Reeves 2017). This will help report what researchers did as many studies do not report adequate detail about non-randomised study (NRS) design. For example, was there a comparison, participant/cluster allocation, what parts of the study were prospective, what variables was comparability between groups assessed.

Two review authors (YBM/AF) will extract data independently. A second review author (ND/ELS) will assess this and if necessary, in the case of any disagreement or discrepancy, a fourth review author will assess this. We will select the fourth review author according to the expertise required to make the relevant decision. Where information is lacking, we will attempt to make contact with study authors.

\section{Assessment of risk of bias in included studies}

Two review authors (ND and YBM) will independently assess the risk of bias potentially introduced by suboptimal design or conduct in each of the included studies. We will resolve disagreements by consensus.

For RCTs we will use the Cochrane 'Risk of bias' tool (Higgins 2017).

We will assess the risk of bias in the domains of selection bias (sequence generation, concealment of allocation), detection bias (blinding of outcome assessors), reporting bias (selective outcome reporting), and attrition bias (incomplete outcome data).

For non-RCTs we will use the ROBINS-I tool (Sterne 2019). We will assess the risk of bias in the domains of pre-intervention (bias due to confounding and selection of participants), at intervention (information bias) and post-intervention (bias due to confounding, selection, information, and reporting).
For any other relevant non-RCT study, such as controlled before and after studies and interrupted time series studies, we will use additional guidance from the Cochrane EPOC group (EPOC 2017).

We will incorporate the results of the 'Risk of bias' assessment into the review through systematic narrative description and commentary about each item.

\section{Measures of treatment effect}

We will report study results organised by mode of enteral tube feeding or other intervention and comparator evaluated. Treatment effects may be measured using dichotomous data, or an ordinal rating scale. For cross-over trials, we will generate, as appropriate, a risk ratio (RR) or mean difference (MD) for pre-crossover results.

- Dichotomous data: for dichotomous data in comparative studies, we will extract or generate RRs and their 95\% confidence intervals (Cls). For primary outcomes, we will calculate using the 'treat-as-one-trial' method, numbers needed to treat for an additional beneficial outcome (NNTB) or numbers needed to treat for an additional harmful outcome (NNTH).

- Continuous data: for effects measured in comparative studies as ordinal data we will treat these as continuous data. We will extract or generate the MD for continuous and ordinal data where the data are provided as a mean and standard deviation (SD). If data are reported pre-intervention and post-intervention, we will report means or proportions for both intervention and control groups and calculate the change from baseline.

- Time-to-event data: we will extract log of the hazard ratios (HRs) and their $95 \% \mathrm{Cls}$. If these are not reported, we will attempt to estimate them from other reported statistics (Parmar 1998). If limitations in the study prevent reporting or generating a HR, we will report the mortality as a dichotomous outcome.

\section{Unit of analysis issues}

In our handling of any included studies, we will consider issues that may impact on findings. For these we will use guidance from the Cochrane Handbook for Systematic Reviews of Interventions (Deeks 2017). These include:

- Groups of participants randomised together with the same intervention (e.g. cluster-randomised trials). These studies will be dealt with on a trial-by-trial basis, depending on the study design.

- Participants receiving more than one intervention (e.g. crossover trials).

- Multiple observations for the same outcomes (such as repeated measures).

\section{Dealing with missing data}

Given the nature of the population, we anticipate a significant amount of missing data as a result of trial or study attrition due to the death of the participant.

We will contact the study authors if there is missing data. For studies using continuous outcomes in which SDs were not reported, and we are unable to calculate the SD from the standard error of the mean (SEM), we will derive the SD from other reported statistics (e.g. Cls, $t$ values, $P$ values). If we are unable to derive the SD, we will follow guidance from the Cochrane Handbook for Systematic Reviews of Interventions and will impute the SDs. 


\section{Assessment of heterogeneity}

We will assess statistical heterogeneity of any combined analysis using the $\mathrm{I}^{2}$ statistic. The $\mathrm{I}^{2}$ statistic is a reliable and robust test to quantify heterogeneity, since it does not depend on the number of trials or on the between-trial variance. The $I^{2}$ statistic measures the extent of inconsistency among trials' results, and can be interpreted as the proportion of total variation in trial estimates that is due to heterogeneity rather than sampling error. We will consider an $1^{2}$ value of greater than $50 \%$ to indicate substantial heterogeneity (Deeks 2017). Where possible, we will undertake subgroup analyses or sensitivity analyses in an attempt to explain heterogeneity.

\section{Assessment of reporting biases}

If there are at least 10 studies included in the analysis, we will prepare funnel plots to estimate small study effects which may represent reporting bias.

\section{Data synthesis}

Where trial data is of sufficient quality and sufficiently similar (in diagnostic criteria, control group, intervention, outcome measure, length of follow-up, and type of analysis), we will combine data in a meta-analysis to provide a pooled effect estimate. We will use a fixed-effect model in the first instance. If we find no statistical heterogeneity, we will use a random-effects model to check the robustness of the fixed-effect model. If there is substantial (over 50\%) statistical heterogeneity, we will report the random-effects model only. Where this occurs, we will state that we used the random-effects model.

We will undertake a descriptive review of the studies that we do not include in the meta-analysis.

\section{Subgroup analysis and investigation of heterogeneity}

Where we identify heterogeneity in a meta-analysis, we plan to undertake subgroup and sensitivity analysis to investigate its possible sources. Subgroup analysis explores whether the overall effect varied with different trial populations, and with the nature and content of the interventions. In this update, we have planned the following subgroup analysis:

- Delivery method (e.g. nasogastric, PEG, or jejunal post-pyloric feeding).

- Type of dementia disease (e.g. Alzheimer's, vascular dementia).

- Clinical setting (e.g. care home or hospital).

We will present investigations of heterogeneity in a table for ease of comparison.

\section{Sensitivity analysis}

If sufficient trials are available, we will seek to perform, in a metaanalysis, sensitivity analyses to explore the influence of:

- Publication status by excluding unpublished trials.

- Trial quality by excluding trials that had a high risk of bias.

\section{GRADE and 'Summary of findings' tables}

Two review authors (a combination of ND; BC; ELS; GR; or YBM) will independently assess the quality of the evidence for all outcomes using the GRADE approach. We will use the guidelines provided in
Chapter 11.2 of the Cochrane Handbook for Systematic Reviews of Interventions (Schünemann 2017).

The GRADE system uses the following criteria for assigning a quality level to a body of evidence (Schünemann 2017).

- High: randomised trials; or double-upgraded observational studies.

- Moderate: downgraded randomised trials; or upgraded observational studies.

- Low: double-downgraded randomised trials; or observational studies.

- Very low: triple-downgraded randomised trials; or downgraded observational studies; or case series/case reports.

The GRADE approach uses five considerations (study limitations, consistency of effect, imprecision, indirectness, and publication bias) to assess the quality of the body of evidence for each outcome. The GRADE system uses the following criteria for assigning grade of evidence.

- High: we are very confident that the true effect lies close to that of the estimate of the effect.

- Moderate: we are moderately confident in the effect estimate; the true effect is likely to be close to the estimate of effect, but there is a possibility that it is substantially different.

- Low: our confidence in the effect estimate is limited; the true effect may be substantially different from the estimate of the effect.

- Very low: we have very little confidence in the effect estimate; the true effect is likely to be substantially different from the estimate of effect.

Factors that may decrease the quality level of a body of evidence are:

- Limitations in the design and implementation of available studies suggesting high likelihood of bias.

- Indirectness of evidence (indirect population, intervention, control, outcomes).

- Unexplained heterogeneity or inconsistency of results (including problems with subgroup analyses).

- Imprecision of results (wide Cls).

- High probability of publication bias (0.7854 to 1.1359$)$.

Factors that may increase the quality level of a body of evidence are:

- Large magnitude of effect.

- All plausible confounding would reduce a demonstrated effect or suggest a spurious effect when results show no effect.

- Dose-response gradient.

We will decrease the grade rating by one $(-1)$ or two (-2) (up to a maximum of -3 to 'very low') if we identify:

- Serious $(-1)$ or very serious $(-2)$ limitations to study quality.

- Important inconsistency $(-1)$.

- Some $(-1)$ or major $(-2)$ uncertainty about directness.

- Imprecise or sparse data (-1).

- High probability of reporting bias $(-1)$. 
In certain circumstances, we will adjust the overall rating for a particular outcome as recommended by GRADE guidelines (Guyatt 2013). For example, we will consider whether there were so few data that the results are highly susceptible to the random play of chance. In other circumstances, we will not downgrade for imprecision if $\mathrm{Cls}$ are wide, if the outcome threshold according to how much harm would be acceptable given a benefit or vice versa.

We will include 'Summary of findings' tables to present the main findings in a tabular format. We will present effect estimates and summarise the quantity and overall certainty of evidence for all primary outcomes and key secondary outcomes in the 'Summary of findings' tables.

\section{ACKN OWLEDGEMENTS}

We thank peer reviewers Joan Teno and a second reviewer (who wishes to remain anonymous) for their comments and feedback.

We also thank consumer reviewer Clare Goodman for her comments and feedback. 


\section{REFERE N C E S}

\section{Additional references}

\section{Alzheimer's Disease International 2009}

Alzheimer's Disease International. World Alzheimer Report 2009. London: Alzheimer's Disease International, 2009.

\section{Alzheimer's Disease International 2015}

Alzheimer's Disease International. World Alzheimer Report 2015: The Global Impact of Dementia. An analysis of prevalence, incidence, cost and trends. London: Alzheimer's Disease International, 2015.

\section{American Geriatrics Society 2014}

American Geriatrics Society Ethics Committee, Clinical Practice and Models of Care Committee. American Geriatrics Society feeding tubes in advanced dementia position statement. Journal of the American Geriatrics Society 2014;62(8):1590-3.

\section{APA 2000}

American Psychiatric Association. Diagnostic and Statistical Manual of Mental Disorders. 4th Edition. Washington DC: American Psychiatric Association, 2000.

\section{Braun 2005}

Braun UK, Rabeneck L, McCullough LB, Urbauer DL, Wray NP, Lairson DR, et al. Decreasing use of percutaneous endoscopic gastrostomy tube feeding for veterans with dementia-racial differences remain. Journal of the American Geriatrics Society 2005;53(2):242-8.

\section{Brooke 2015}

Brooke J, Ojo O. Enteral nutrition in dementia: a systematic review. Nutrients 2015;7(4):2456-68.

\section{Browne 2017}

Browne J, Edwards DA, Rhodes KM, Brimicombe DJ, Payne RA. Association of comorbidity and health service usage among patients with dementia in the UK: a population-based study. BMJ Open 2017;7(3):e012546.

\section{Bunn 2014}

Bunn F, Burn AM, Goodman C, Rait G, Norton S, Robinson L, et al. Comorbidity and dementia: a scoping review ofthe literature. BMC Medicine 2014;12:192.

\section{Chang 2016}

Chang WK, Lin KT, Tsai CL, Chung CH, Chien WC, Lin CS. Trends regarding percutaneous endoscopic gastrostomy: a nationwide population-based study from 1997 to 2010. Medicine 2016;95(24):e3910.

\section{Cintra 2014}

Cintra MT, de Rezende NA, de Moraes EN, Cunha LC, da Gama Torres HO. A comparison of survival, pneumonia, and hospitalization in patients with advanced dementia and dysphagia receiving either oral or enteral nutrition. Journal of Nutrition, Health \& Aging 2014;18(10):894-9.

\section{Ciocon 1988}

Ciocon JO, Silverstone FA, Graver LM, Foley CJ. Tube feedings in elderly patients: indications, benefits, and complications. Archives of Internal Medicine 1988;148(2):429-33.

\section{Davies 2014}

Davies N, Maio L, Rait G, lliffe S. Quality end-of-life care for dementia: what have family carers told us so far? A narrative synthesis. Palliative Medicine 2014;28(7):919-30.

\section{Davies 2016}

Davies N, Mathew R, Wilcock J, Manthorpe J, Sampson E, Lamahewa $\mathrm{K}$, et al. A co-design process developing heuristics for practitioners providing end of life care for people with dementia. BMC Palliative Care 2016;15:68.

\section{Davies 2017}

Davies N, Rait G, Maio L, Iliffe S. Family caregivers' conceptualisation of quality end-of-life care for people with dementia: a qualitative study. Palliative Medicine 2017;31(8):726-33.

\section{Davies 2018}

Davies N, Manthorpe J, Sampson EL, Lamahewa K, Wilcock J, Mathew R, et al. Guiding practitioners through end of life care for people with dementia: the use of heuristics. PLOS One 2018;13(11):e0206422.

\section{De 2019}

De $\mathrm{D}$, Thomas $\mathrm{C}$. Enhancing the decision-making process when considering artificial nutrition in advanced dementia care. International Journal of Palliative Nursing 2019;25(5):216-23.

\section{Deeks 2017}

Deeks JJ, Higgins JPT, Altman DG (editors), on behalf of the Cochrane Statistical Methods Group. Chapter 9: Analysing data and undertaking meta analyses. In: Higgins JP, Churchill R, Chandler J, Cumpston MS, editor(s), Cochrane Handbook for Systematic Reviews of Interventions version 5.2.0 (updated June 2017). The Cochrane Collaboration, 2017. Available from www.training.cochrane.org/handbook. Cochrane.

\section{EPOC 2017}

Cochrane Effective Practice, Organisation of Care (EPOC). What study designs can be considered for inclusion in an EPOC review and what should they be called? 2017. EPOC Resources for review authors. epoc.cochrane.org/resources/epoc-resourcesreview-authors (accessed 8 March 2019).

\section{Finucane 1996}

Finucane TE, Bynum JP. Use of tube feeding to prevent aspiration pneumonia. Lancet 1996;348(9039):1421-4.

\section{Gil 2018}

Gil E, Agmon M, Hirsch A, Ziv M, Zisberg A. Dilemmas for guardians of advanced dementia patients regarding tube feeding. Age and Ageing 2018;47(1):138-43. 


\section{Guyatt 2013}

Guyatt G, Eikelboom JW, Akl EA, Crowther M, Gutterman D, Kahn SR, et al. A guide to GRADE guidelines for the readers of JTH. Journal of Thrombosis and Haemostasis 2013;11(8):1603-8.

\section{Higgins 2017}

Higgins JPT, Altman DG, Sterne JAC (editors). Chapter 8: Assessing risk of bias in included studies. In: Higgins JP, Churchill R, Chandler J, Cumpston MS, editor(s), Cochrane Handbook for Systematic Reviews of Interventions version 5.2.0 (updated June 2017). The Cochrane Collaboration, 2017. Available from www.training.cochrane.org/handbook. Cochrane.

\section{Hoefler 2000}

Hoefler JM. Making decisions about tube feeding for severely demented patients at the end of life: clinical, legal, and ethical considerations. Death Studies 2000;24(3):233-54.

\section{Johnston 2008}

Johnston SD, Tham TCK, Mason M. Death after PEG: results of the national confidential enquiry into patient outcome and death. Gastrointestinal Endoscopy 2008;68(2):223-7.

\section{Kai 2015}

Kai K, Hashimoto M, Amano K, Tanaka H, Fukuhara R, Ikeda M. Relationship between eating disturbance and dementia severity in patients with Alzheimer's disease. PLOS One 2015;10(8):e0133666.

\section{Livingston 2015}

Livingston G, Frankish H. A global perspective on dementia care: a Lancet Commission. Lancet 2015;386(9997):933-4.

\section{Mathew 2016}

Mathew R, Davies N, Manthorpe J, Iliffe S. Making decisions at the end of life when caring for a person with dementia: a literature review to explore the potential use of heuristics in difficult decision-making. BMJ Open 2016;6(7):e010416.

\section{Matthews 2013}

Matthews FE, Arthur A, Barnes LE, Bond J, Jagger C, Robinson L, et al. A two-decade comparison of prevalence of dementia in individuals aged 65 years and older from three geographical areas of England: results of the Cognitive Function and Ageing Study I and II. Lancet 2013;382(9902):1405-12.

\section{Mitchell 2016}

Mitchell SL, Mor V, Gozalo PL, Servadio JL, Teno JM. Tube feeding in US nursing home residents with advanced dementia, 2000-2014. Journal of the American Medical Association 2016;316(7):769-70.

\section{NICE 2018}

National Institute for Health and Care Excellence (NICE). Dementia: assessment, management and support for people living with dementia and their carers. London: NICE, 2018.

\section{Odom 2003}

Odom S, Barone J, Docimo S, Bull S, Jorgensson D. Emergency department visits by demented patients with malfunctioning feeding tubes. Surgical Endoscopy and Other Interventional Techniques 2003;17(4):651-3.

\section{Papachristou 2017}

Papachristou I, Hickey G, lliffe S. Dementia informal caregiver obtaining and engaging in food-related information and support services. Dementia 2017;16(1):108-18.

\section{Parmar 1998}

Parmar MK, Torri V, Stewart L. Extracting summary statistics to perform meta-analyses of the published literature for survival endpoints. Statistics in Medicine 1998;17(24):2815-34.

\section{Punchik 2018}

Punchik B, Komissarov E, Zeldez V, Freud T, Samson T, Press Y. Doctors' knowledge and attitudes regarding enteral feeding and eating problems in advanced dementia. Dementia and Geriatric Cognitive Disorders Extra 2018;8(2):268-76.

\section{Rait 2010}

Rait G, Walters K, Bottomley C, Petersen I, Iliffe S, Nazareth I. Survival of people with clinical diagnosis of dementia in primary care: cohort study. BMJ 2010;341:c3584.

\section{Reeves 2017}

Reeves BC, Wells GA, Waddington H. Quasi-experimental study designs series-paper 5: a checklist for classifying studies evaluating the effects of health interventions-a-taxonomy without labels. Journal of Clinical Epidemiology 2017;89:30-42.

\section{Reeves 2019}

Reeves BC, Deeks JJ, Higgins JPT, Shea B, Tugwell P, Wells GA. Chapter 24: Including non-randomized studies on intervention effects. In: Higgins JPT, Thomas J, Chandler J, Cumpston M, Li T, Page MJ, Welch VA (editors). Cochrane Handbook for Systematic Reviews of Interventions version 6.0 (updated July 2019). Cochrane, 2019. Available from www.training.cochrane.org/ handbook. London: Cochrane.

\section{Regnard 2010}

Regnard C, Leslie P, Crawford H, Matthews D, Gibson L. Gastrostomies in dementia: bad practice or bad evidence?. Age and Ageing 2010;39(3):282-4.

\section{Reisberg 1994}

Reisberg B, Sclan SG, Franssen E, Kluger A, Ferris S. Dementia staging in chronic care populations. Alzheimer Disease and Associated Disorders 1994;8(Suppl 1):S188-205.

\section{Sampson 2018}

Sampson EL, Candy B, Davis S, Gola AB, Harrington J, King M, et al. Living and dying with advanced dementia: a prospective cohort study of symptoms, service use and care at the end of life. Palliative Medicine 2018;32(3):668-81.

\section{Schünemann 2017}

Schünemann HJ, Oxman AD, Higgins JP, Vist GE, Glasziou P, Akl E, et al. on behalf of the Cochrane GRADEing Methods Group and the Cochrane Statistical Methods Group. Chapter 11: Completing 'Summary of findings' tables and grading the confidence in or quality of the evidence. In: Higgins JP, Churchill 
R, Chandler J, Cumpston MS, editor(s), Cochrane Handbook for Systematic Reviews of Interventions version 5.2.0 (updated June 2017). The Cochrane Collaboration, 2017. Available from www.training.cochrane.org/handbook. Cochrane.

\section{Sleeman 2019}

Sleeman KE, de Brito M, Etkind S, Nkhoma K, Guo P, Higginson IJ, et al. The escalating global burden of serious health-related suffering: projections to 2060 by world regions, age groups, and health conditions. Lancet Global Health 2019;7(7):e883-92.

\section{Snyder 2013}

Snyder EA, Caprio AJ, Wessell K, Lin FC, Hanson LC. Impact of a decision aid on surrogate decision-makers' perceptions of feeding options for patients with dementia. Journal of the American Medical Directors Association 2013;14(2):114-8.

\section{Sterne 2019}

Sterne JAC, Hernán MA, McAleenan A, Reeves BC, Higgins JPT. Chapter 25: Assessing risk of bias in a non-randomized study. In: Higgins JPT, Thomas J, Chandler J, Cumpston M, Li T, Page MJ, Welch VA (editors). Cochrane Handbook for Systematic Reviews of Interventions version 6.0 (updated July 2019). Cochrane, 2019. Available from www.training.cochrane.org/handbook. London: Cochrane.

\section{Teno 2012}

Teno JM, Gozalo P, Mitchell SL, Kuo S, Fulton AT, Mor V. Feeding tubes and the prevention or healing of pressure ulcers. Archives of Internal Medicine 2012;172(9):697-701.

\section{van der Steen 2014}

van der Steen JT, Radbruch L, Hertogh CM, de Boer ME, Hughes JC, Larkin P, et al. White paper defining optimal palliative care in older people with dementia: a Delphi study and recommendations from the European Association for Palliative Care. Palliative Medicine 2014;28(3):197-209.

\section{Volkert 2015}

Volkert D, Chourdakis M, Faxen-Irving G, Frühwald T, Landi F, Suominen $\mathrm{MH}$, et al. ESPEN guidelines on nutrition in dementia. Clinical Nutrition 2015;34(6):1052-73.

\section{Watt 2019}

Watt AD, Jenkins NL, McColl G, Collins S, Desmond PM. Ethical issues in the treatment of late-stage Alzheimer's disease. Journal of Alzheimer's Disease 2019;68(4):1311-6.

\section{WHO 1993}

World Health Organization. The ICD-10 Classification of Mental and Behavioural Disorders: Diagnostic Criteria for Research. Geneva: WHO, 1993.

\section{WHO 2012}

World Health Organization (WHO). Dementia: a Public Health Priority. Geneva: WHO, 2012.

\section{WHO 2018}

World Health Organization. International classification of diseases for mortality and morbidity statistics (11th Revision). 2018 version. https://icd.who.int/browse11/l-m/en (accessed 11 November 2019).

\section{WHO 2019}

World Health Organization (WHO). Dementia fact sheet. www.who.int/news-room/fact-sheets/detail/dementia. Geneva: WHO, (accessed 02 December 2019).

\section{Xie 2008}

Xie J, Brayne C, Matthews FE, Medical Research Council Cognitive Function and Ageing Study collaborators. Survival times in people with dementia: analysis from population based cohort study with 14 year follow-up. BMJ 2008;336(7638):258-62.

\section{References to other published versions of this review Sampson 2008}

Sampson EL, Candy B, Jones L. Enteral feeding for older people with advanced dementia. Cochrane Database of Systematic Reviews 2008, Issue 3. [DOI: 10.1002/14651858.CD007209]

\section{Sampson 2009}

Sampson EL, Candy B, Jones L. Enteral tube feeding for older people with advanced dementia. Cochrane Database of Systematic Reviews 2009, Issue 2. [DOI: 10.1002/14651858.CD007209.pub2]

\section{APPENDICES}

\section{Appendix 1. MEDLINE search strategy}

1. dement\$..mp.

2. alzheimer\$.mp.

3. dementia/

4. alzheimer disease/

5. enteral nutrition/

6. nutritional support/

7. percutaneous feeding.ti,ab.

8. artificial feeding.ti,ab.

9. artificial hydration.ti,ab.

10. endoscopic gastrostomy.ti,ab.

11. tube feeding.ti,ab.

12. peg.ti,ab. 
13. enteral feeding.ti,ab.

14. stomach tube\$.ti,ab.

15. forced feeding.ti,ab.

16. percutaneous feeding.ti,ab.

17. artificial nutrition.ti,ab.

18. nutritional support.ti,ab.

19. enteral nutrition.ti,ab.

20. feeding methods.ti,ab.

21. (tube adj2 (nasogastric OR naso-jejunal OR jejunostomy post-pyloric feeding)).ti,ab.

22. or $/ 1-4$

23. or $/ 5-21$

24. 21 and 22

25. (control adj2 (group or groups or patient ${ }^{\star}$ or cohort $\left.{ }^{\star}\right)$ ).ti,ab.

26. (controlled adj study).ti,ab.

27. comparative study.ti,ab.

28. clinical trial/

29. multicenter study/

30. "before-and-after".ti,ab.

31. CBA.ti,ab.

32. Interrupted Time Series Analysis/

33. Interrupted time series.ti,ab.

34. ("non-random?sed trial" or "non-random?sed stud*").ti,ab.

35. ("nonrandom?sed trial*" or "nonrandom?sed stud*").ti,ab.

36. Controlled Before-After Studies/

37. pragmatic clinical trial.pt.

38. (quasiexperiment ${ }^{\star}$ or quasi experiment ${ }^{\star}$ or pseudo experiment ${ }^{\star}$ or pseudoexperiment $^{\star}$ ).ti,ab.

39. ((pretest or pre test) and (posttest or post test)).ti,ab.

40. repeated measur*.ti,ab.

41. randomised controlled trial/

42. controlled clinical trial/

43. randomly.ab.

44. groups.ab.

45. placebo.ab.

46. or $/ 25-45$

47.24 and 46

\section{CONTRIBUTIONS OFAUTHORS}

All authors contributed to the conception and design of this protocol.

All authors contributed to drafting the protocol, commented on it critically for intellectual content, and approved the final version for publication.

\section{DECLARATIONS OF INTEREST}

Nathan Davies: none known.

Yolanda Barrado-Martin: none known.

Greta Rait: none known.

Akiko Fukui: none known.

Bridget Candy: none known.

Christina H Smith: none known.

Jill Manthorpe: none known.

Kirsten J Moore: none known.

Elizabeth L Sampson: none known.

Victoria Vickerstaff: none known. 


\section{SOURCES OF SUPPORT}

\section{Internal sources}

- Marie Curie, UK.

This work was supported by Marie Curie [grant number MCRGS-20171219-8004]. ELS's and BC's posts are supported by Marie Curie core grant MCCC-FCO-16-U.

- Alzheimer's Society, UK.

ND was supported by a Junior Fellowship from Alzheimer's Society, UK (grant number 325: AS-JF-16b-012).

KM was supported by a Senior Fellowship from Alzheimer's Society, UK (grant number 325: AS-SF-16-004).

\section{External sources}

- NIHR, UK.

This protocol was supported by the National Institute for Health Research (NIHR), via Cochrane Infrastructure funding to the Cochrane Dementia and Cognitive Improvement group. The views and opinions expressed therein are those of the authors and do not necessarily reflect those of the Systematic Reviews Programme, the NIHR, the National Health Service, or the Department of Health 